\title{
APPLICABILITY OF HEAVY ION BEAM PROBING FOR STELLARATOR W7-X
}

S. PERFILOV, A. MELNIKOV*, L. KRUPNIK ${ }^{1}$, H.J. HARTFUSS ${ }^{2}$

RRC "Kurchatov Institute”, Moscow, Russia

${ }^{1}$ NNC "KhIPT", Kharkov, Ukraine

${ }^{2}$ IPP Max-Plank Institute, Greifswald, Germany

Pages: 12 .

Figures: 11. 


\begin{abstract}
The feasibility study for HIBP on the stellarator W7-X was done to provide the measurements of the radial profiles of plasma potential, density and their fluctuations. Calculations of probing $\mathrm{Tl}^{+}$beam trajectories were done for the various $\mathrm{W} 7-\mathrm{X}$ magnetic configurations with $B_{\text {tor }}=2.5 \mathrm{~T}$. They show that satisfactory access is possible for K11-N11 port combination. The trajectory optimization aiming for the maximal plasma observation was done for the chosen port combination.
\end{abstract}




\section{INTRODUCTION}

\section{A. Basic Principles}

Heavy Ion Beam Probe (HIBP) is a unique method to measure the plasma potential and thus the radial electric field in the core of hot magnetized plasmas [1]. When the beam of highenergy singly charged ions passes through the plasma, some of the injected beam ions undergo a secondary ionization predominantly by plasma electrons. The ionization takes place along the full beam path in the plasma. Because of their higher charge state, the secondary ions deviate from the primary beam trajectory and form a broad fan of ions leaving the plasma (Fig. 1). The secondary ions that enter the detector aperture, originate from a small part of the primary beam in the plasma, called the sample volume, which has typical dimensions of $(0.1-1) \mathrm{cm}^{3}$. Under some conditions it can be even less. The energy difference between the secondary ions leaving the plasma and the primary ions is equal to the electric potential $\varphi$ at the sample volume. The intensity of the secondary beam reflects the electron density $n_{\mathrm{e}}$ in the sample volume. The toroidal velocity of the secondary beam in the detector reflects the poloidal component of magnetic vector potential (poloidal magnetic field or plasma current density).

By redirecting the probing beam with electrostatic sweep plates, we can rapidly change the position of the sample volume. The position of the sample volume can also be changed shot to shot by changing the energy of the primary particles. HIBP has a continuous signal in time, which provides a high temporal resolution, limited by the acquisition electronics.

\section{B. OBJECTIVES OF THE W-7X HIBP PROJECT}

The goal of the W-7X HIBP project is to provide the plasma potential measurements in a reasonably wide plasma area, which can give various contributions for the $\mathrm{W}-7 \mathrm{X}$ scientific program. The main emphasis is the radial profile of the plasma electric potential. With the measurements of plasma electric potential (mean profiles and fluctuations) HIBP can contribute 
to the physical understanding of edge (H-mode) and internal transport barriers (ITB). The density profile can also be reconstructed from the secondary beam intensity profile.

\section{C. W-7X FEATURES (DIFFICULTIES FOR HIBP)}

W-7X presents a complex case for HIBP implementation. The relatively low $B_{t o r}$ could make it possible to use a "low" beam energy for the probing. However, the machine design with large toroidal coils and the high level of the stray magnetic field claims produce "long" trajectories located far outside the plasma area which are more difficult to optimize at "low" beam energies. That, in turn, necessitates the use of additional beam control hardware in the beamlines for the primary and the secondary beams adjustment and control.

The 3D nature of the W7-X magnetic field leads to the quite non-planar (3D) structure of the beam trajectories. The $2 \mathrm{MeV}$ ion accelerator was already procured for W7-X HIBP, so the beam energy is limited. The port combination $(\mathrm{K} 11+\mathrm{N} 11)$ was also already selected at the previous stage of the project. The port design brings the geometric limitations for the beam trajectories location. The divertor assembly brings the additional limitation for the trajectories.

\section{3D TRAJECTORY CALCULATIONS}

The trajectory calculation code solves the $3 \mathrm{D}$ motion equation for the probing

particle $m \frac{\partial \vec{v}}{\partial t}=q e \vec{E}+q e[\vec{v} \times \vec{B}]$

where vector $\boldsymbol{B}$ is the full magnetic field of the device. The trajectory calculations were done neglecting the plasma electric field $\boldsymbol{E}$. For the W-7X, the $\mathrm{Tl}^{+}$beam was chosen, $\mathrm{m}=205$. The standard HIBP scheme with the basic secondary ionization reaction was assumed - electron impact ionization, producing the secondary ions:

$$
\mathrm{Tl}^{+}+\mathrm{e}=\mathrm{Tl}^{++}+2 \mathrm{e}
$$


So, for the primary beam $q=1$ and for the secondary particles $q=2$. The primary trajectories were originated at the Injection point, located far outside the ports. The secondary trajectories were started from the primary one in the plasma area. They are followed until the Detection point, located far outside the output port. This point may be considered as an entrance slit of the energy analyzer.

\section{A. Magnetic field model}

Trajectory calculations were made for the standard W-7X configurations with $B_{t o r}=2.5 \mathrm{~T}$. The full 3D structure of the magnetic field was calculated by W7-X magnetic field code (Gourdon code). The spatial calculation grid has a spacing of $10 \mathrm{~cm}$ while the angular step was 1 deg. For a more dense grid, smaller steps were chosen: $5 \mathrm{~cm}$ and $0.5 \mathrm{deg}$. An interpolation procedure was used for the determination of magnetic field components in the desired point of the area of calculations. The components of the magnetic field were found as a combination of the fields generated by each filament, which were calculated by the Biot-Savart law. The filaments positions and currents were taken for the standard configuration [2].

\section{B. Control electric field model}

The electric field, generated by the each pair of control (sweeping) parallel plates is simulated separately. For each pair of plates the full Laplace equation is solved with the finite element method. This means that we don't distinguish the control region and the fringe regions. The calculation shows that the electric potential is less than $10^{-6}$ of its source (plates) value at some distance, which is a few times larger than the plates size. Out of this area the electric potential was neglected. Kinetic energy conservation is verified after the particle leaves the $E$ field calculation area. The accuracy of energy conservation was better than $10^{-6}$. 
There were two pairs of the plates modeled for the primary beam control in toroidal and poloidal directions. Both are located outside the machine port. The secondary beamline plates will be modeled at future stages of the project development.

\section{C. Secondary fan structure}

The general character of the secondary fan structure is shown in Fig. 2 in an example of from the MAST tokamak [3]. The primary trajectory (fat curve) starts in injector port of the vacuum chamber. The secondary fan consists of two parts. The quasi-parallel fan (thin solid curves) covers the detector port from up to down when the birth point moves forward with the primary probing particle. This is the conventional case ' $A$ '. Some of secondaries intersect in a focus point, because the toroidal field works as a magnetic lens. If the detector is located behind the focus, the intersected fan covers the port in the opposite direction from down to up, when the birth point moves forward with the primary particle. This is the unusual case ' $\boldsymbol{B}$ '. If the detector is located before the focus, we get the case ' $\boldsymbol{A}$ ' again. The most general case is the combination of both cases. Two areas can be united if we put the detector in the position ' $C$ '. Particles ionized in different sample volumes on the same primary trajectories (case $\boldsymbol{A}+\boldsymbol{B}$ ) may come to the detector with different toroidal deflections. Therefore, the natural way to separate them is to vary the initial toroidal angle. For the case ' $C$ ' we are not able to separate two parts of the united sample volume. In this case we have to accept comparatively poor space resolution, about $10 \mathrm{~cm}$ or more. The empirical observation of the related phenomenon of the sample volume divergence was done at the gas calibtration experiments on JIPPT-IIU tokamak outside the plasma region [4].

\section{D. Preliminary calculations}

The verification of the trajectories with the one obtained in an earlier study was done as the initial step of the work. The lower port is used for the initial beam injection. The results of 
the preliminary calculations are presented on Fig. 3. The coincidence of the trajectories shows a good agreement with the results obtained in [5]. In Fig. 3 one may see the important features of the secondary fan for the W7-X: 1) it is close to planar, but not exactly planar, 2) it has $\gamma$-shape in the cross-section. The latter means that not the single, but two trajectories, ' $A$ ' and ' $\boldsymbol{B}$ ' type, born in different plasma regions may come to the detector point simultaneously.

\section{THE SCHEME OPTIMIZED TO PREVENT THE INTERSECTION BETWEEN PROBING TRAJECTORIES AND DIVERTOR ASSEMBLY}

\section{A. The optimal probing scheme.}

At first, the infinitesimally thin initial beam was modeled. The probing scheme was found to get the maximal extent of the observation area in the plasma and was not affected by the divertor assembly, Fig. 4. It was suggested to locate energy analyzer far outside the machine port. The position of the intermediary Detection point D is chosen near the outer flange of the N11 port. The passage from the detector point to the energy analyzer will be done with the secondary beamline. In this work we will not discuss the details of the secondary beamline. We will focus our considerations of the trajectories up to the intermediary detection point.

For the chosen detector point position both A and B cases are realized. Let us call the secondary trajectories as type A and type B correspondingly. They are denoted in different colors in the figure 4, type A - in light gray, type B- in dark gray.

\section{B. Radial reference of the detector line $\left(B_{\text {tor }}=2.5 \mathrm{~T} ; E_{\text {beam }}=2 \mathrm{MeV}\right)$}

Figure 5 presents the location of the sample volume for the optimal scheme. All trajectories are passing through the ports K11 and N11 without any loss. The gap between the trajectories and divertor assembly is about a dozen $\mathrm{cm}$.

Figure 6 shows the radial location of the sample volumes versus sweeping voltage. It is possible to observe the outer half of plasma radius with type 'B' trajectories $(0.5<\rho<1.0)$. All the 
trajectories of type ' $\mathbf{A}$ ' are born in a very limited inner radial area $(0.1<\rho<0.15)$. The intermediate area $(0.15<\rho<0.5)$ belongs to trajectories of ' $\mathbf{C}$ ' type with very poor spatial resolution even for ideal beam with zero width.

To reach the very center of the plasma with the optimal probing scheme, one need to reduce slightly the toroidal magnetic field up to $B_{t o r}=2.43 \mathrm{~T}$. In this case the trajectories of type 'A' will reach the plasma axis.

\section{THE TRAJECTORIES CALCULATIONS MODELLING THE REAL BEAM DIMENSIONS}

The sample volume splitting and existance of the ' $\mathrm{C}$ ' zone cause the substantial increase in the sample volume (deterioration of the spatial resolution).

For the finite beam calculations we have fixed a $2 \mathrm{~cm}$ beam width. The beam is modelled with a set of 100 beam filaments. The Gaussian beam current distribution was modelled. The sample volumes for zone ' $\boldsymbol{B}$ ' and the corresponding radii are presented in Fig. 7. It is assumed that the secondaries from zone ' $A$ ' were removed by the collimation. Open circles denote the length of the sample volume along the primary trajectory, $\lambda$. Let us introduce the characteristic for the radial resolution, the radial length of the sample volume $\lambda_{\rho}$, expressed as a fraction of fraction of $\boldsymbol{a}_{\text {seperatrix }} . \lambda_{\rho}$ is denoted in open triangles in Fig. 7. The solid line denotes the radius of the centre of mass for the sample volume. In the interval from $-7 \mathrm{kV}$ to approximately $-4 \mathrm{kV}$ of $U_{\text {sweep }}(0.85<\rho<1.0)$, some part of the secondary beam, reaching the detector, was born outside the plasma border. That is the reason for the decrease of $\lambda$ observed as the sample volume moves to the plasma border ( $U_{\text {sweep }}$ tends to $\left.-4 \mathrm{kV}\right)$.

In the interval from $-9 \mathrm{kV}$ to approximately $-7 \mathrm{kV}$ of $U_{\text {sweep }}(0.6<\rho<0.85)$ there are no beam losses. In principle, this is a good area for the measurements. One can see that the radial 
resolution, $\lambda_{\rho}$, lies within the interval $0.3<\lambda_{\rho}<0.5$ for this area. Such space resolution is extremely poor.

In the interval from $-12 \mathrm{kV}$ to approximately $-9 \mathrm{kV}$ of $U_{\text {sweep }}(0.3<\rho<0.6)$ there are some losses of the secondaries born at several filaments. Filaments loss starts from peripheral filaments at $U_{\text {sweep }}=-12 \mathrm{kV}$. At the final point $U_{\text {sweep }}=-12 \mathrm{kV}$ all of them are gone.

We can conclude that the radial resolution, $\lambda_{\rho}$, is unacceptably high for this probing scheme.

\section{THE STUDY AIMING TO IMPROVE THE SPATIAL RESOLUTION FOR THE STANDARD CONFIGURATION}

We discussed the features of the optimized probing scheme in the previous section. We note a rather limited observation area for this scheme with rather poor spatial resolution (see Figs. 6, 7).

To improve the spatial resolution, the optimized scheme was modified. The main idea of the trajectories modification was to separate as much as possible the ' $C$ ' zone from the area of measurements. The possible means were found to vary the distance between the Injection and Detection points. One should has take into account the geometrical limitations due to port size and the divertor assembly location. A realistic beam diameter of $d=1 \mathrm{~cm}$ was used for the modeling.

\section{A. The use of the A-type trajectories}

To observe the detector line formed with ' $A$ ' type trajectories, the Injection point was shifted up. To let the trajectories pass through the ports, we have to reduce the beam energy to $E_{\text {beam }}=$ 1.5 MeV, see Fig 8 . 
The probing scheme parameters are: $X_{\mathrm{inj}}=422.6 \mathrm{~cm}, Y_{\mathrm{inj}}=129.2 \mathrm{~cm}, Z_{\mathrm{inj}}=223 \mathrm{~cm}, X_{\mathrm{det}}=$ $232.7 \mathrm{~cm}, Y_{\text {det }}=281.7 \mathrm{~cm}$, and $Z_{\text {det }}$ is not fixed. The trajectories do not intersect the divertor assembly. Note that the total radial observation interval becomes shorter (compare to Fig. 7).

Fig 9 presents the spatial resolution for the discussed scheme. Open triangles denote $\lambda_{\rho}$ (radial length of the sample volume as a fraction of $\boldsymbol{a}_{\text {seperatrix }}$ ) for $d_{\text {beam }}=10 \mathrm{~mm}$. Black solid line - the radius of the centre of mass for the sample volume. Open circles denote the length of the sample volume along the primary trajectory, $\lambda$. Shown is the averaged $\lambda$ value over all the beam filaments.

The $\boldsymbol{C}$-zone $\left(-15 \mathrm{kV}<U_{\text {sweep }}<-12 \mathrm{kV}\right)$ manifests itself by drastic increase of $\lambda$ and the $\lambda_{\rho}$. In the interval from $--10 \mathrm{kV}$ to approximately $+20 \mathrm{kV}$ of $U_{\text {sweep }}(0.48<\rho<0.95)$, which can be used for the measurements, there are no any beam losses. In principle, this is the good area for the measurements. One can see that $\lambda_{\rho}$ lies within the interval $0.01<\lambda_{\rho}<0.02$. This is quite small in comparison with the case described in Fig.7, which is $0.3<\lambda_{\rho}<0.5$ for $0.6<\rho<0.85$. The disadvantage of this case is a rather wide interval of the sweeping voltage. Finally, the spatial resolution is reasonably acceptable and the observation area is extended. However, observation area still remains too small, it is located at the outer part of the plasma column .

Note that some part of the secondary beam, reaching the detector, was born outside the plasma border. That is the reason for the decrease of $\lambda_{\rho}$ curve, when sample volume moves to the plasma border $\left(20 \mathrm{kV}<U_{\text {sweep }}<30 \mathrm{kV}\right)$.

\section{B. Using of the B-type trajectories}

To observe the detector line formed with trajectories of $\boldsymbol{B}$ type, the Detection point was shifted up. It is not necessary to reduce the beam energy to let the trajectories pass through the ports in this case. So we keep the maximal $\boldsymbol{E}_{\text {beam }}=2.0 \mathrm{MeV}$. 
The probing scheme parameters are: $X$ inj $=422.6 \mathrm{~cm}, Y i n j=124.2 \mathrm{~cm}, Z$ inj $=223 \mathrm{~cm}, X$ det $=232.7 \mathrm{~cm}, Y$ det $=230 \mathrm{~cm}$, and Zdet is not fixed. The trajectories do not intersect the divertor assembly, see Fig 10.

Fig. 11 presents the spatial resolution for the discussed scheme. Open triangles denote $\lambda_{\rho}$ (radial length of the sample volume as a fraction of $\boldsymbol{a}_{\text {seperatrix }}$ ) for $d_{\text {beam }}=1 \mathrm{~cm}$. Black solid line the radius of the centre of mass for the sample volume. Open circles is the length of the sample volume along the primary trajectory, $\lambda$. Shown is the averaged value over all the beam filaments. Note that the total radial observation interval becomes shorter (compare to Fig. 7).

The $\boldsymbol{C}$-zone is seen clearly in the interval $\left(-1.5 \mathrm{kV}<U_{\text {sweep }}<+1.5 \mathrm{kV}\right)$. In the interval from $+1.5 \mathrm{kV}$ to approximately $+7.5 \mathrm{kV}$ of $U_{\text {sweep }}(0.45<\rho<0.95)$, which can be used for the measurements, there are no beam losses. Again, this is a good area for measurements. One can see that the integration interval is $0.12<\lambda_{\rho}<0.16$. This is quite a large value in comparison with the case described in Fig. 9, but smaller than the case, described in Fig. 7. The advantage of the discussed case is rather narrow interval of the sweeping voltage.

Finally, the obtained spatial resolution looks to be marginally acceptable. The observation area is the same as for A-type trajectories.

Similar to the A-type trajectories, some part of the secondary beam, reaching the detector, was born outside the plasma border. That is the reason for the decrease of the triangles denoted curve, as sample volume moves to the plasma border $\left(6 \mathrm{kV}<U_{\text {sweep }}<9 \mathrm{kV}\right)$.

\section{CONCLUSIONS}

1. The optimized probing scheme for K11-N11 port combination was found.

2. This scheme allows us to avoid intersection between beam trajectory and divertor plates assembly. 
3. This probing scheme $\{2.5 \mathrm{~T}, 2 \mathrm{MeV}\}$, provides maximal sample volume penetration into plasma column $0.08<\rho<1.0$, but unacceptably poor radial resolution.

4. The sample volume splitting takes place for the analyzed port combination. To select the desired trajectories, secondary beam collimation is needed.

5. There was another probing scheme found for the study of the outer parts of plasma minor radius: $0.48<\rho<1.0$, for $\{2.5 \mathrm{~T}, 1.5 \mathrm{MeV}\}$. The spatial resolution $0.012<\lambda_{\rho}<0.015$ is quite good for $1 \mathrm{~cm}$ beam diameter. The peripheral trajectories are rather close (at the range of beam diameter) to the ports walls. Very fine trajectory is required for this scheme to avoid beam loss at the port wall.

\section{Acknowledgements}

We appreciate the collaboration with Dr Harmeyer, Dr Kisslinger and Dr Neuner. The activity of the Kurchatov team has been supported by INTAS Grant No 2001-2056 and RFBR Grant 05-02-17016.

\section{REFERENCES}

1. IEEE Trans. on the Plasma Science, August 1994. The whole HIBP special issue.

2. T. ANDREEVA, "Vacuum Magnetic Configurations of Wendelstein 7-X." Preprint IPP III/270, May 2002.

3. A.V. MELNIKOV and S.V. PERFILOV, "Effect of the sample volume splitting in heavy ionbeam probing (HIBP) for spherical tokamaks (by example of mega ampere spherical tokamak)" Rev. Sci. Instrum. 70, 21402 (1999).

4. Y. HAMADA, A. NISHIZAWA, Y.KAWASUMI, et. al. "Measurements of profiles of the space potential in JIPP T-IIU tokamak plasmas by slow poloidal and fast toroidal sweepsof heavy ion beam", Plasma Phys. Control. Fusion, 361743 (1994). 
5. A. TEUBEL, "Orbit Calculations for the Determinations of the Heavy Ion Beam Probe Ports on W7X.” Preprint IPP III/214, November 1996. 


\section{Figure captions}

Fig. 1. HIBP basic principles.

Fig. 2. The principles of HIBP sample volume splitting (by example of MAST HIBP)

Fat black trajectory - the primary trajectory. A, B and C denote the various locations of the detection point. Thin gray lines - secondaries of type 'A', reaching detector in all locations. Thin black trajectories - secondaries of type 'B', reaching the $\mathrm{B}$ and $\mathrm{C}$ detector positions only.

Fig 3. Reference to the earlier calculations.

Thin black trajectories - the primaries and secondaries for the previous calculations (Courtesy of

Dr. U. Neuner). Thick black trajectory - the primary trajectory, started at Injection point I. Gray trajectories - the full secondary fan for the given energy and the initial position I.

Fig.4. The trajectories for $\{2.5 \mathrm{~T}, 2 \mathrm{MeV}\}$ (maximal penetration) probing scheme.

The black line - primary trajectory in the K11, started at Injection point I. Dark gray tubes - port walls, gray plates - diverter, light gray trajectories - secondaries type $\boldsymbol{A}$, dark gray trajectories type $\boldsymbol{B}$ ones. Thick black trajectory - primary trajectory leaving the plasma. D - detection point for the secondaries.

Fig.5. The sample volumes for $\{2.5 \mathrm{~T}, 2 \mathrm{MeV}\}$ (maximal penetration) probing scheme.

Short gray pieces - sample volumes on the detector line.

Fig.6. Radial reference versus $U_{\text {sweep }}$ (injection angle).

Fig.7. "Finite beam" calculation results. $E_{\text {beam }}=2.0 \mathrm{MeV}, d_{\text {beam }}=2 \mathrm{~cm}$, type B trajectories.

Fig.8. The side view of the trajectories with $E_{\text {beam }}=1.5 \mathrm{MeV}$.

Fig.9. "Finite beam" calculation results $E_{\text {beam }}=1.5 \mathrm{MeV}, d_{\text {beam }}=1 \mathrm{~cm}$, type A trajectories.

Fig 10. The side view of the trajectories with $E_{\text {beam }}=2.0 \mathrm{MeV}$.

Fig.11. "Finite beam" calculation results $E_{\text {beam }}=2.0 \mathrm{MeV}, d_{\text {beam }}=1 \mathrm{~cm}$, type B trajectories. 
Figures

\section{Basic principles}

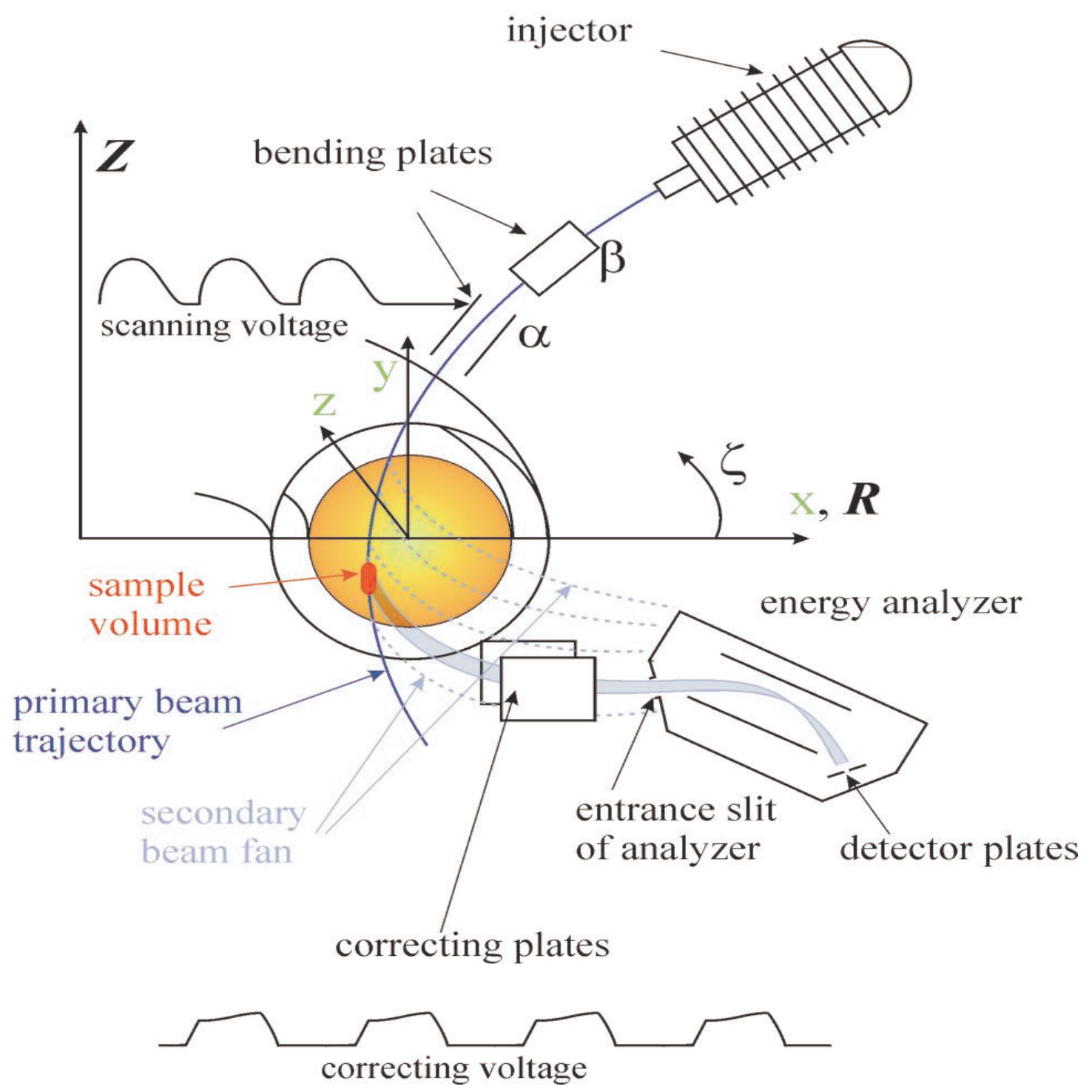

Fig.1 
MAST

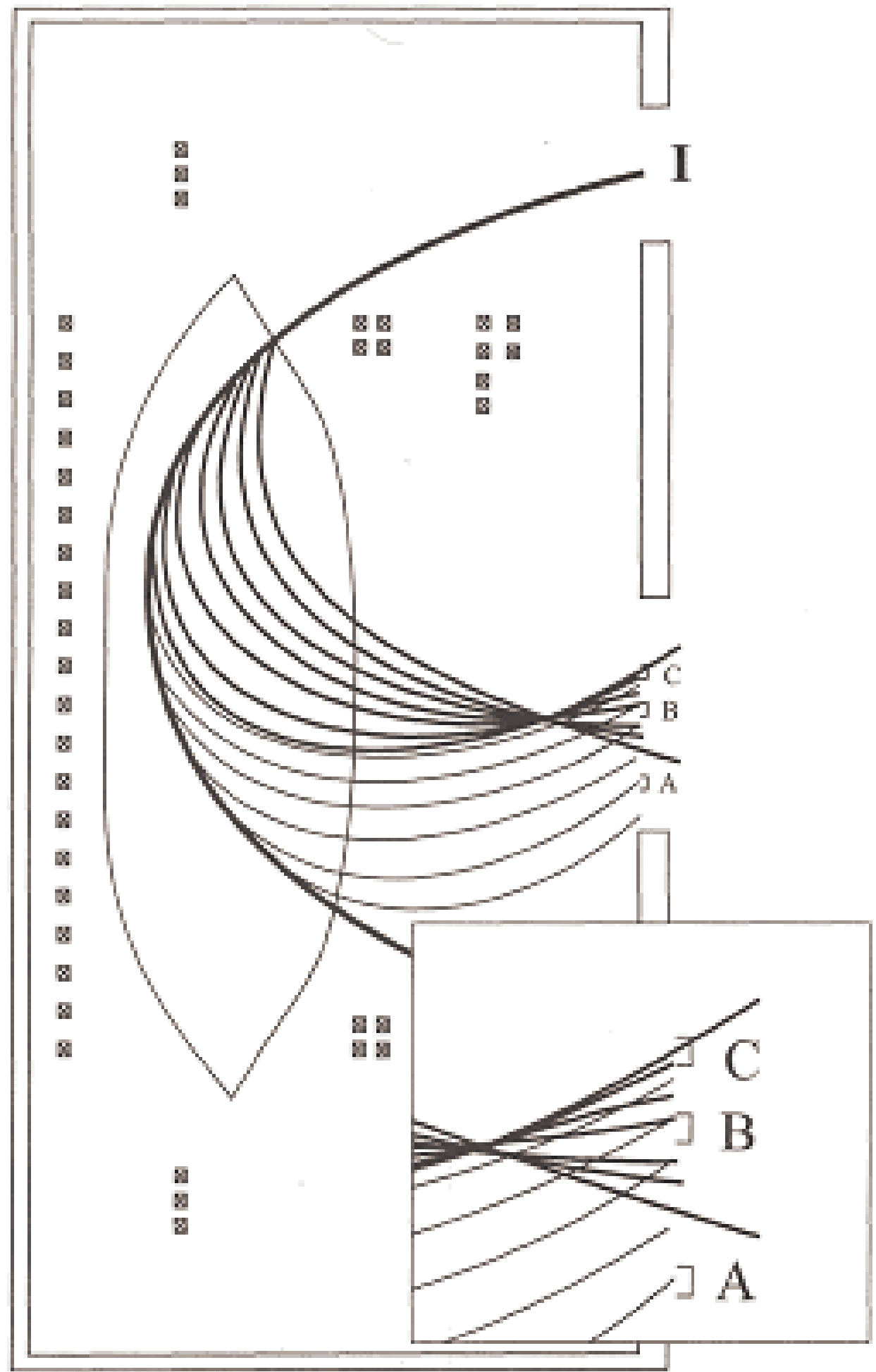

Fig.2 


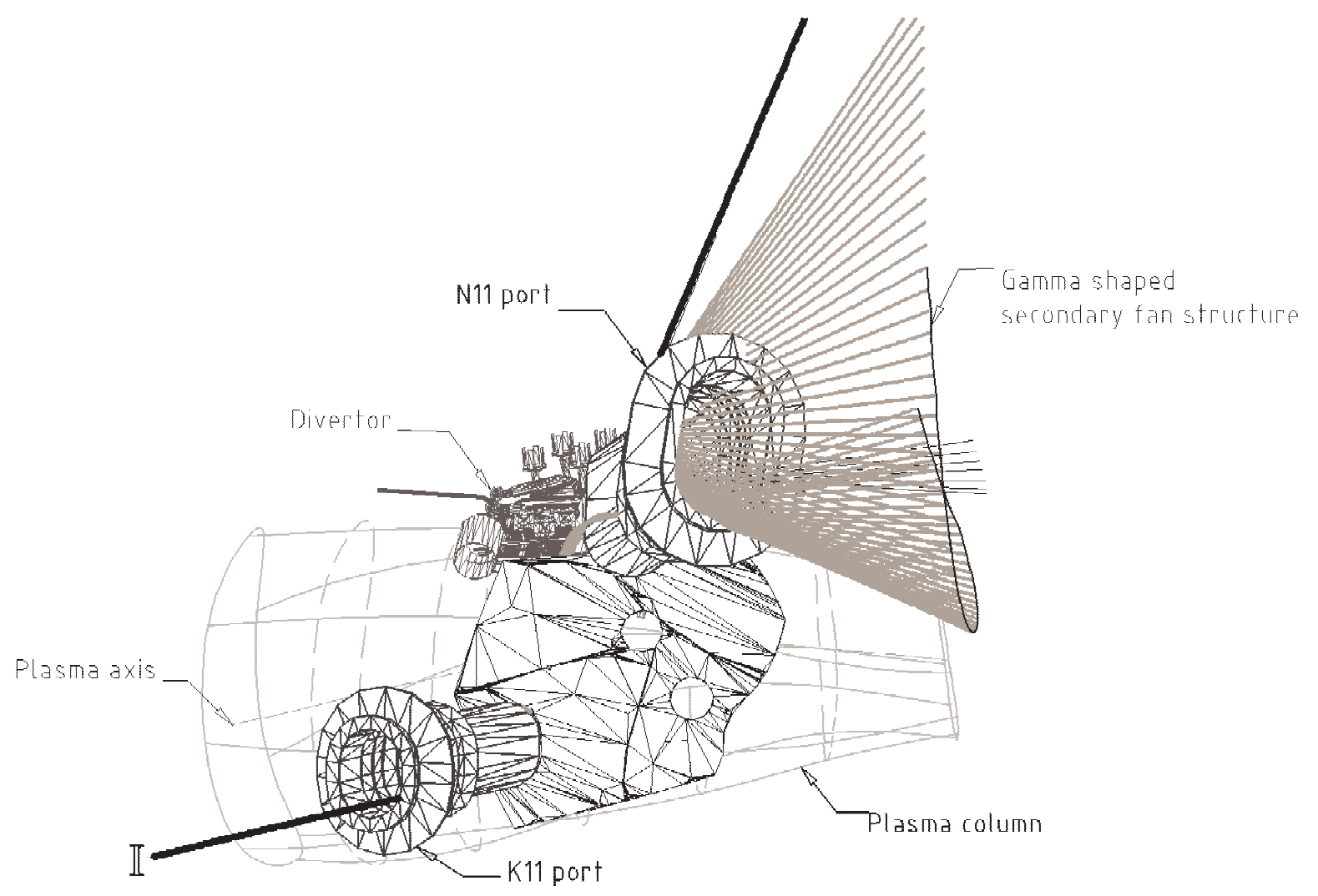

Fig. 3 


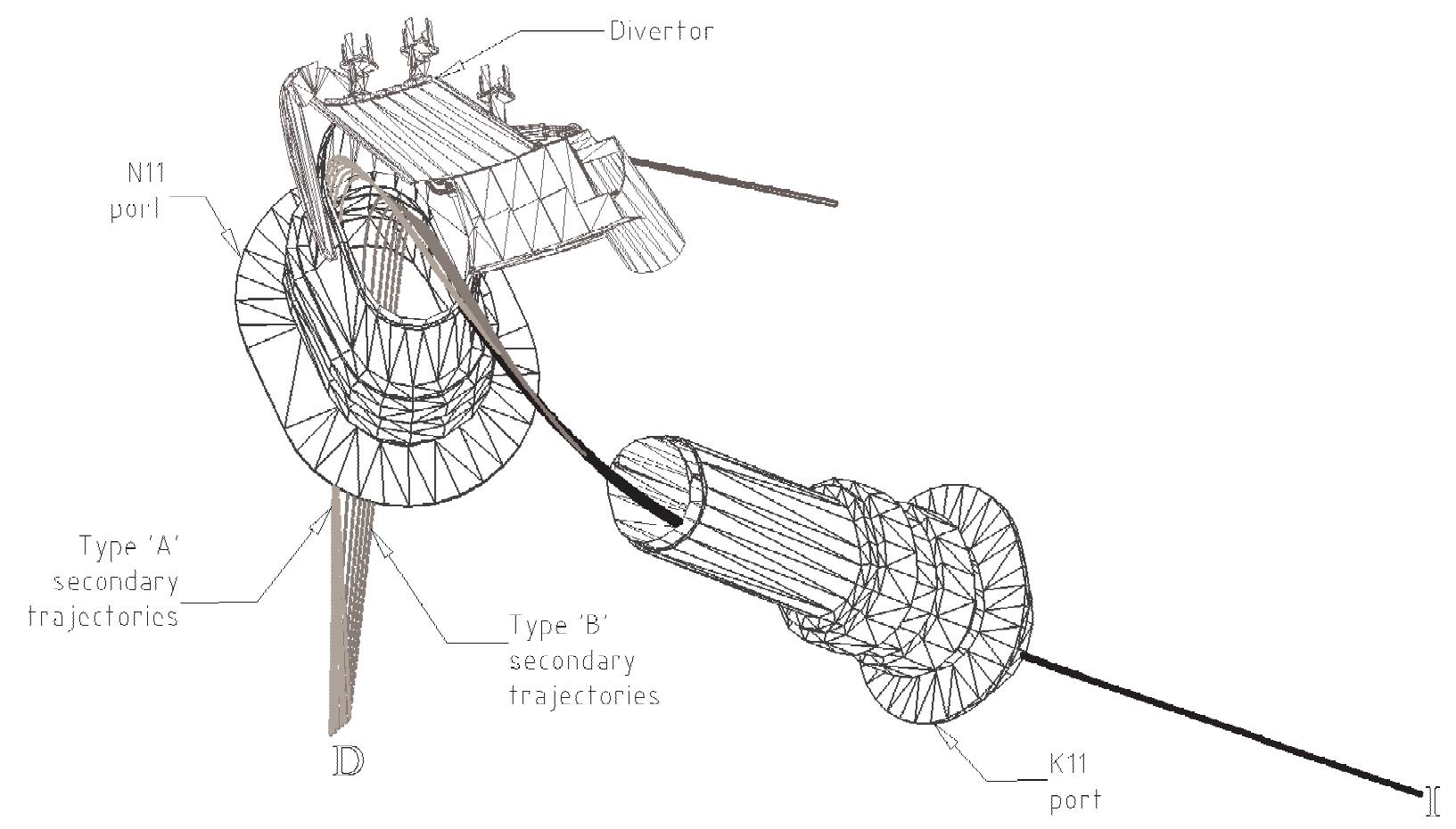

Fig.4 


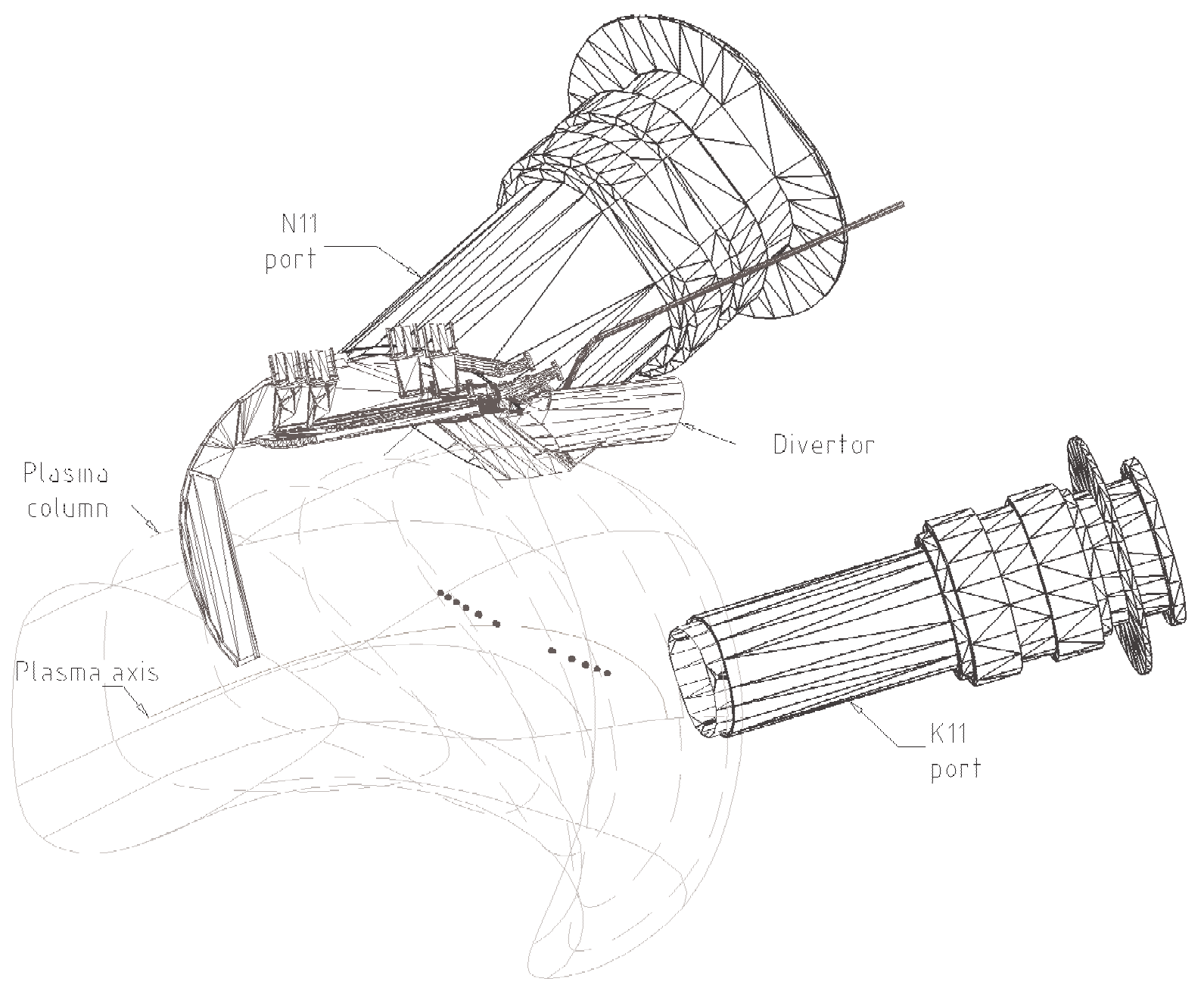

Fig.5 


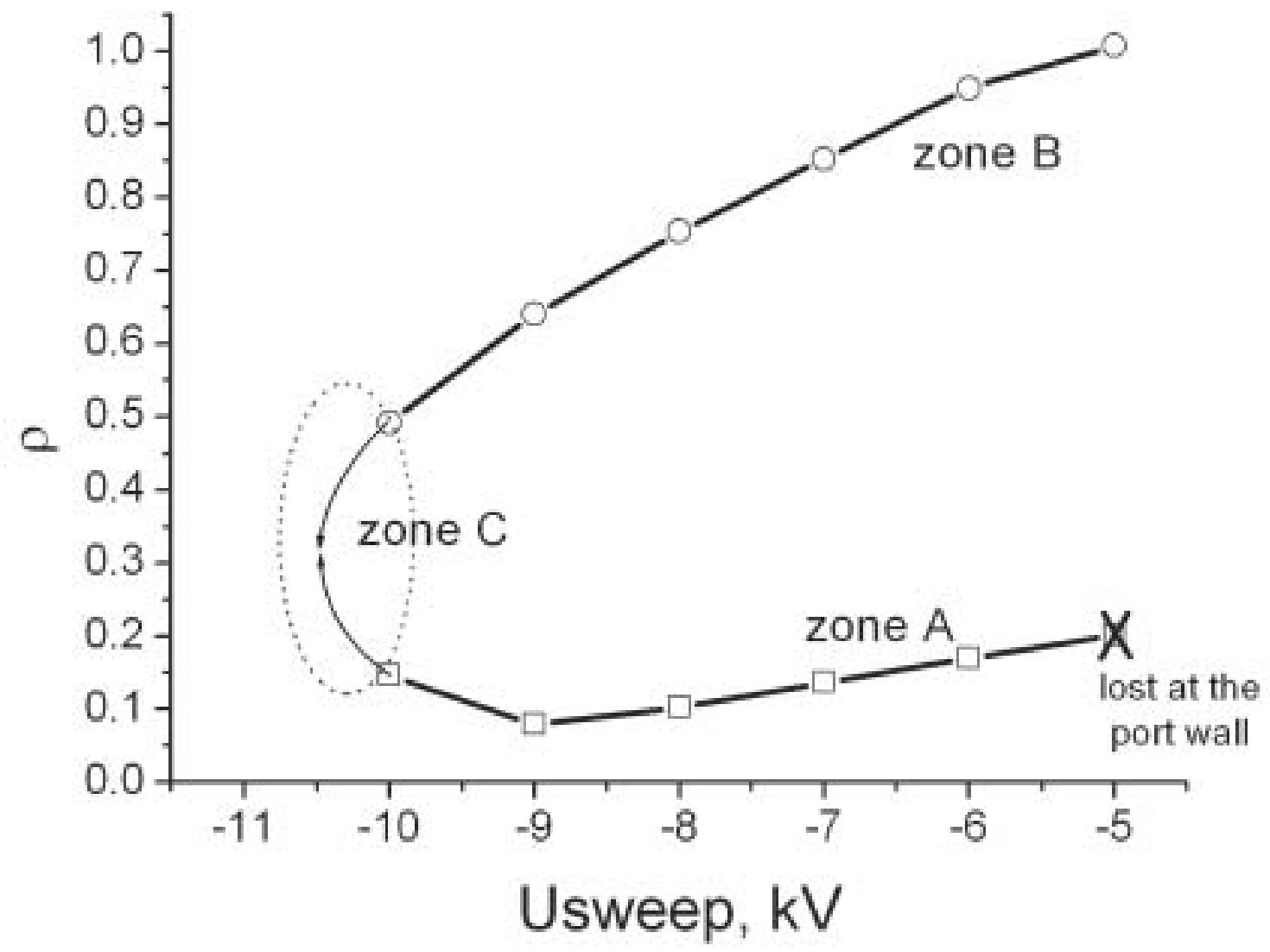

Fig.6 


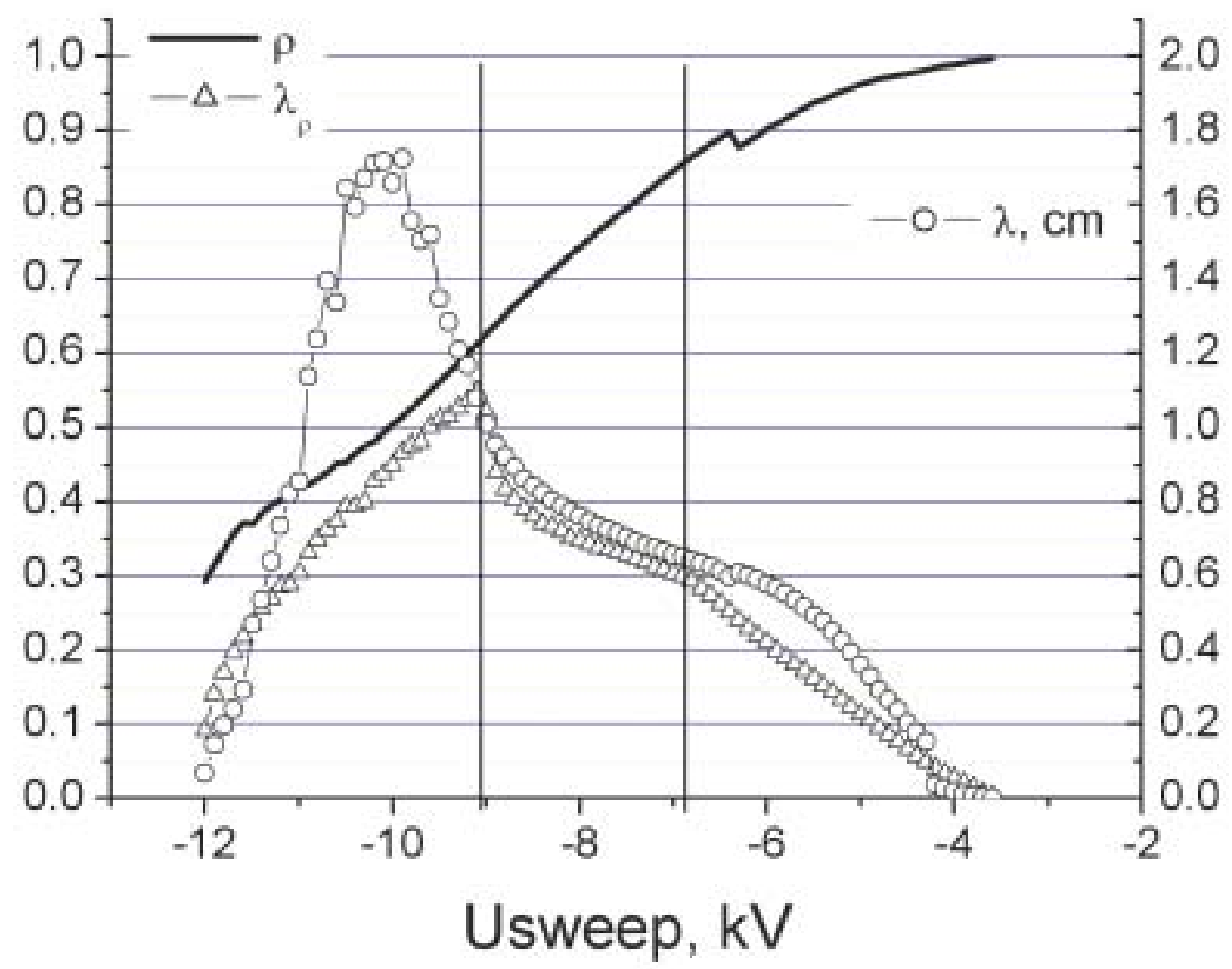

Fig.7 


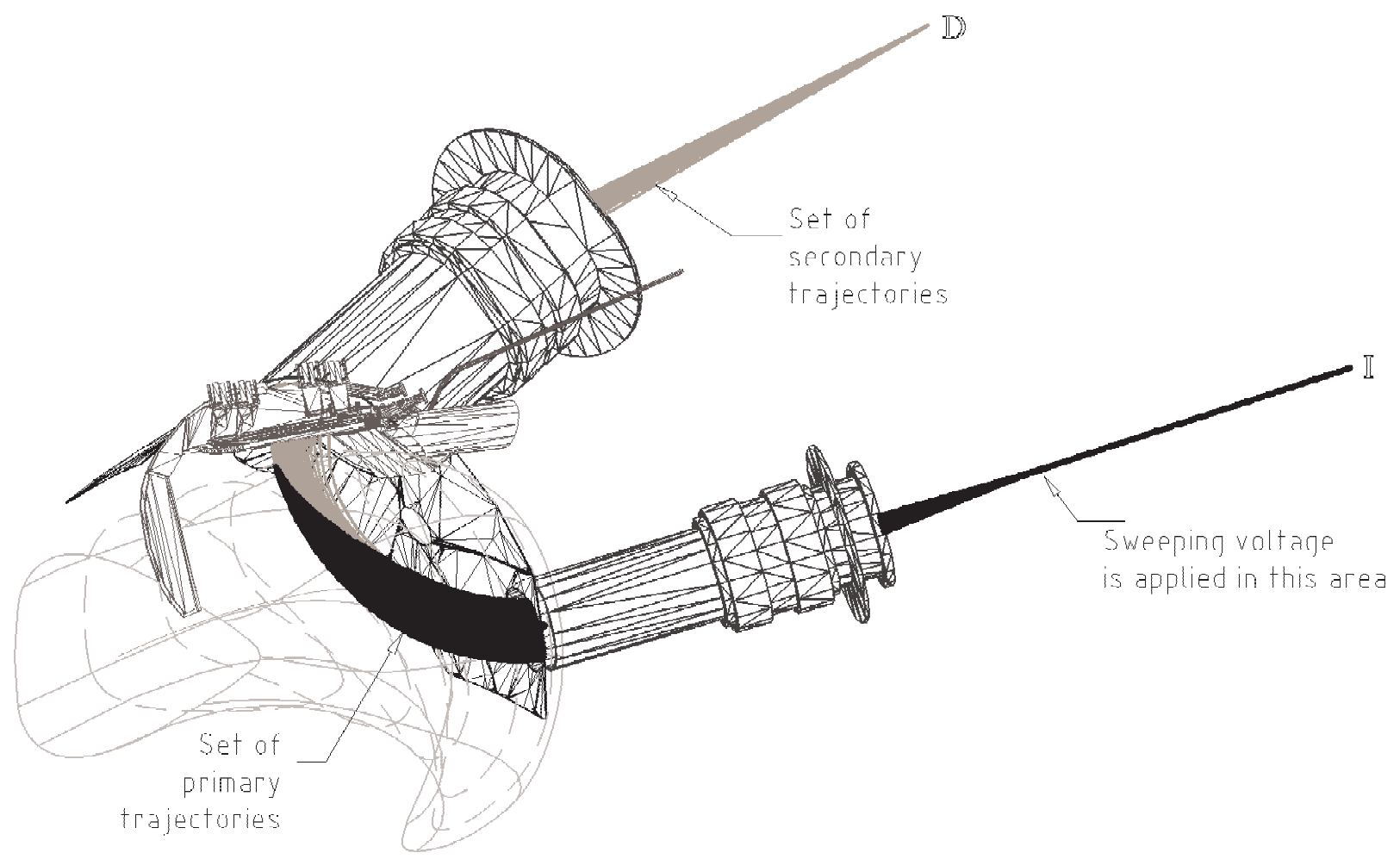

Fig.8 


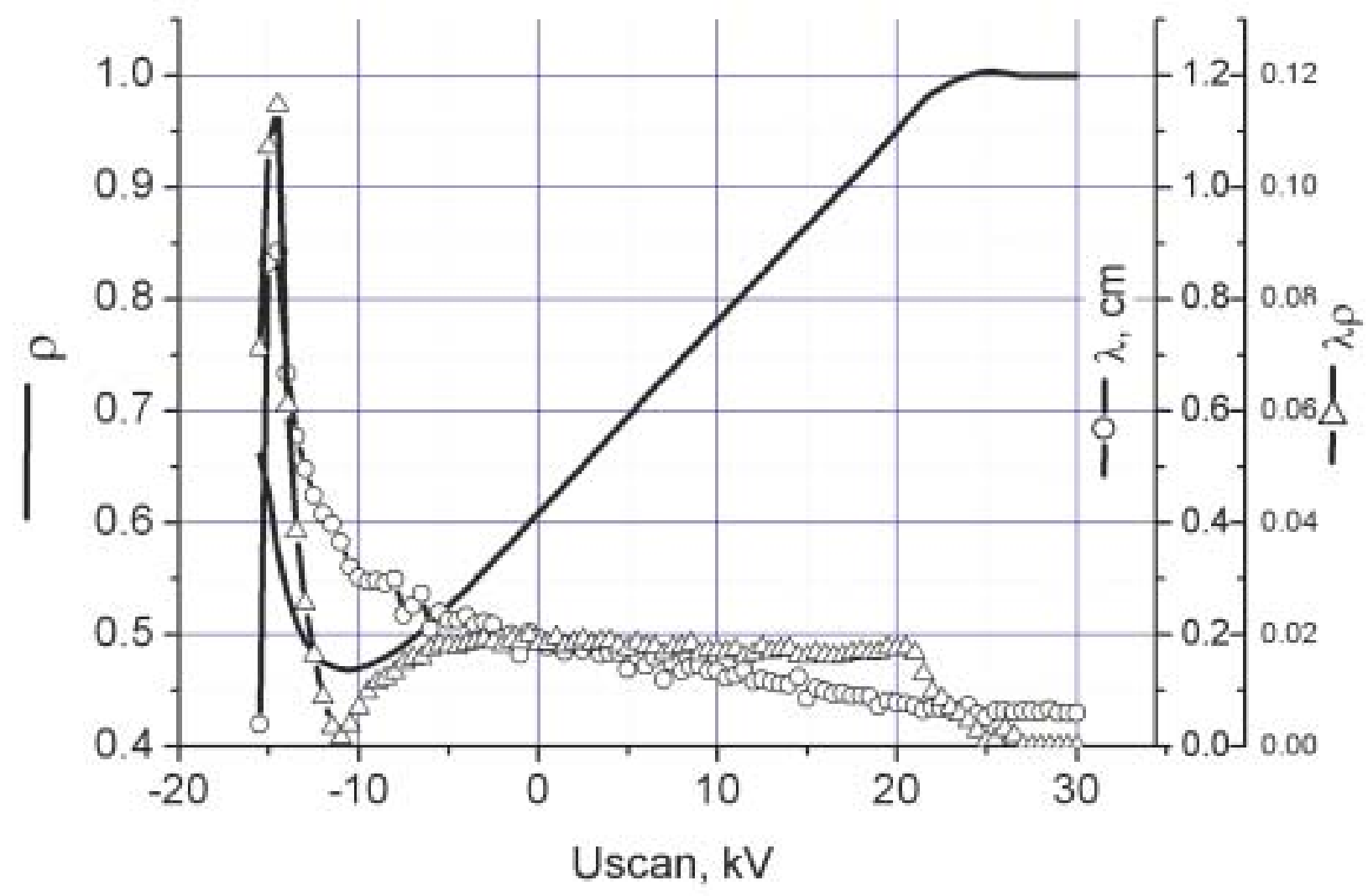

Fig. 9 


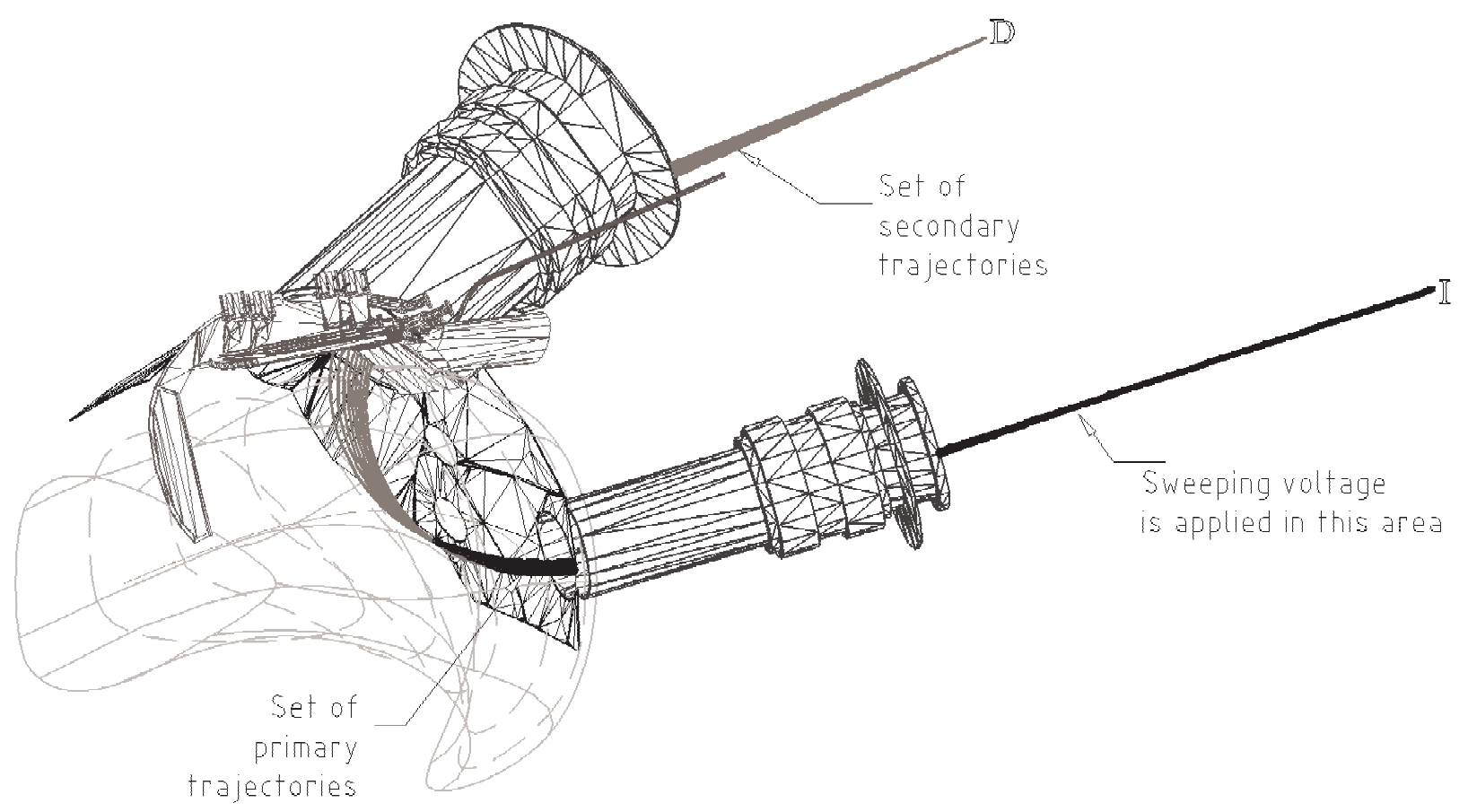

Fig. 10. 


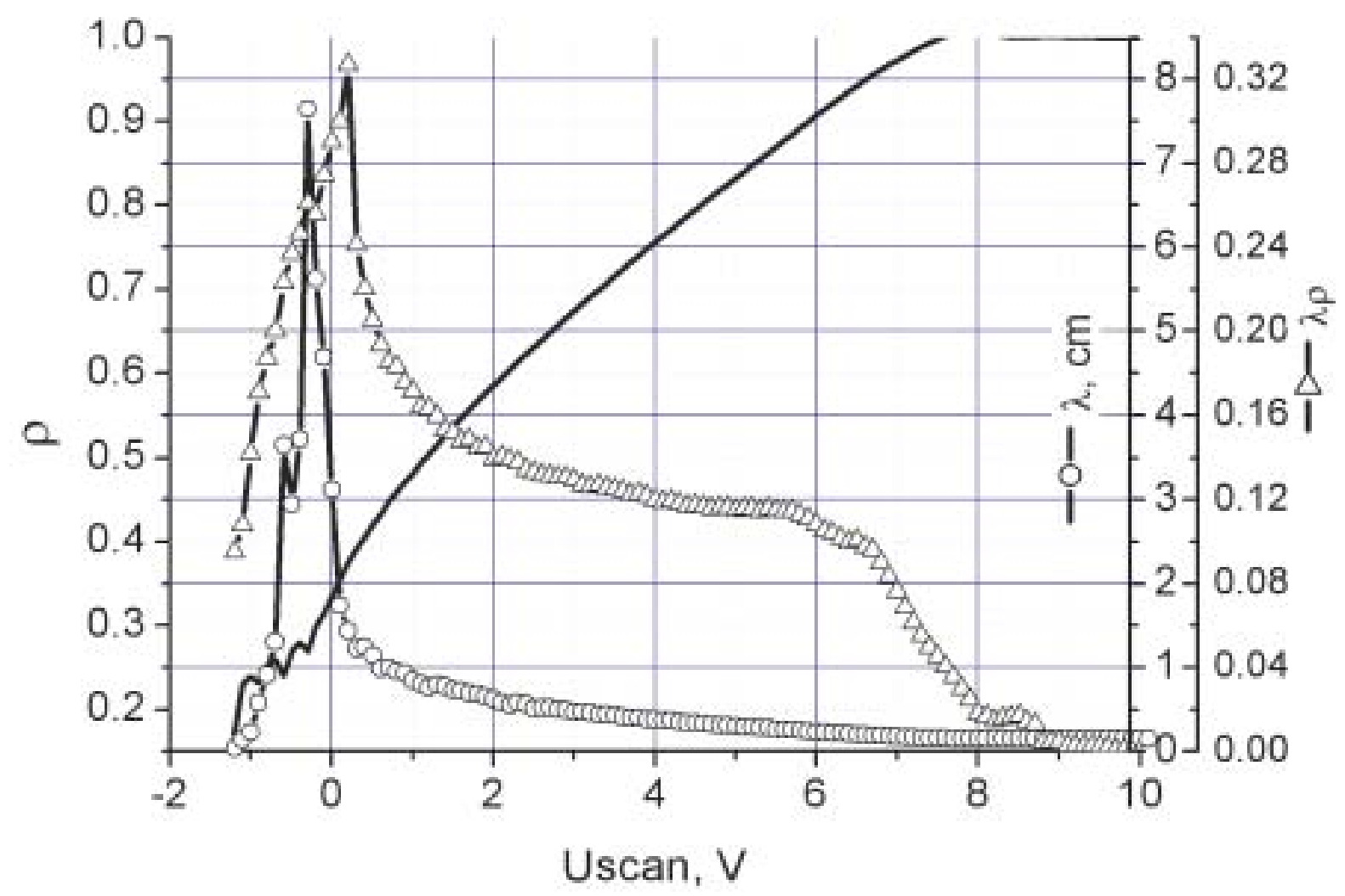

Fig. 11 